\title{
Avances en las Mejoras de Procesos Software en las MiPyMEs Desarrolladoras de Software: Una Revisión Sistemática
}

\author{
Gerzon E. Gómez, Antonio A. Aguileta, Grisel B. Ancona, Omar S. Gómez \\ Facultad de Matemáticas \\ Universidad Autónoma de Yucatán \\ Mérida, México \\ gerzon.gomez@uady.mx, aaguilet@uady.mx,berenis_ancona@hotmail.com,omar.gomez@uady.mx
}

\begin{abstract}
Resumen - Este trabajo presenta la aplicación de un protocolo para revisiones sistemáticas de Ingeniería de Software. En este artículo el protocolo es utilizado como un modelo formal aplicado a la búsqueda de publicaciones relacionadas con las adaptaciones SPI llevadas a cabo en MiPyMEs desarrolladoras de software, en el período comprendido de 1995 a diciembre de 2013, centrándose en tendencias, países, y sectores que publican, así como en los modelos, metodologías, estándares y procesos de soporte del software del área de calidad. Los resultados obtenidos sugieren que en la comunidad de Ingeniería de Software hay un interés creciente en este tema, por ejemplo, la mayoría de las investigaciones realizadas surgen en el sector educativo. El modelo de procesos y la metodología más utilizada es CMMi y Xtreme Programing, respectivamente. El estándar más utilizado es el ISO/ IEC 15504 y el proceso de soporte del software del ciclo de vida del software mayormente abordado es SQA.
\end{abstract}

Palabras Clave-MiPyME, revisión sistemática, mejora del proceso software, calidad del software.

\section{INTRODUCCIÓN}

La micro, pequeña y mediana empresa (MiPyME) productora de software ha cobrado importancia en el mundo debido a que favorece el crecimiento de las economías nacionales [1] y a que representa el 99\% (véase Tabla 1) de las empresas de software, de las cuales las micro (menos de 10 empleados) representan el $78 \%$, las pequeñas (de 10 a menos de 50 empleados) representan el $16 \%$ y las medianas (de 50 a menos de 250 empleados) representan el 5\% [2]. No obstante, dicha importancia de la MiPyME desarrolladora de software, puede verse opacada por sus problemas particulares, tales como el tiempo de entrega de sus productos software, la calidad esperada y el presupuesto asignado $[3,4]$.

En la actualidad se han desarrollado un conjunto de modelos, estándares y metodologías como CMM [5], ISO/IEC 15504 [6] que ayudan a las organizaciones a mejorar la calidad de sus procesos y productos, así como sus proyectos [6,7]. No obstante, debido a las características particulares de la MiPyME, tales como: presupuestos limitados, carencia de personal especializado, infraestructura inadecuada así como reservas financieras limitadas [8]. El establecimiento de estos modelos y metodologías, dado el contexto de las MiPyMEs, debiera realizarse de un modo particular y visiblemente diferente a como se hace en organizaciones de mayor tamaño. Además esto no es tan sencillo como el hecho de considerar los programas de mejora como versiones a escala de las grandes compañías [9], por lo que las MiPyMEs desarrolladoras de software requieren prácticas eficientes de Ingeniería de Software adaptadas a su tamaño y tipo de negocio [2].
En este artículo se presenta una revisión sistemática de la literatura acerca del tópico: adaptaciones en la mejora del proceso software (SPI) en las MiPyMEs, en el período comprendido de 1995 a diciembre de 2013, con el fin de presentar información actualizada sobre las tendencias de este tópico como son: países y sectores que abordan el tema, así como modelos, metodologías, estándares, y procesos de soporte del área de calidad reportados en este tipo de empresas. Esta revisión sistemática se centra en aportaciones reportadas sobre procesos de soporte del área de calidad del ciclo de vida del software. Otras revisiones similares, tales como: Pino et al. [10] se enfoca en las aportaciones que se han realizado en los procesos del ciclo de vida del software; Dybå y Dingsøyr [11] que se enfoca en los beneficios y limitaciones de las metodologías ágiles; Von Wangenheim et al. [12] orientada a la revisión de modelos de capacidad y madurez del proceso de software; o Duron et al. [13] enfocada en conocer cómo se realiza la implementación de la mejora de procesos software.

TABLA 1. Porcentaje de EMPRESAs MiPyMEs DesarRolladoras de SOFTWARE A NIVEL MUNDIAL. (ADAPTADO DE FAYAD ET AL. [2]).

\begin{tabular}{|c|c|c|c|c|c|}
\hline $\begin{array}{c}\text { Microem- } \\
\text { presas }\end{array}$ & $\begin{array}{c}\text { Peque- } \\
\text { nas }\end{array}$ & $\begin{array}{c}\text { Media- } \\
\text { nas }\end{array}$ & $\begin{array}{c}\text { Gran- } \\
\text { des }\end{array}$ & Total & MiPyMEs \\
\hline $78 \%$ & $16 \%$ & $5 \%$ & $1 \%$ & $100 \%$ & $99 \%$ \\
\hline
\end{tabular}

Una revisión sistemática de la literatura permite identificar, evaluar, interpretar y sintetizar un conjunto de investigaciones existentes y relevantes en un tema de interés particular. Este tipo de revisiones se ejecutan de forma rigurosa e imparcial para que tengan un alto valor científico. La principal motivación para emprender una revisión sistemática es incrementar la posibilidad de detectar más resultados reales en el tema de interés que los que pueden ser detectados con revisiones de menor dimensión.

Este trabajo está organizado de la siguiente manera: La sección II presenta de manera general el método para revisiones sistemáticas. En la sección III se describe la aplicación del protocolo de revisiones sistemáticas. En la sección IV se describe la extracción de la información. La sección $\mathrm{V}$ presenta el resumen de resultados. Finalmente la sección VI muestra las conclusiones de este trabajo.

\section{DESCRIPCIÓN DEL MÉTODO PARA REVISIONES SISTEMÁTICAS}

\section{A. ¿Qué es la Revisión sistemática?}

El término "Revisión Sistemática" (RS) es usado para referirse a una metodología específica de investigación, desarrollada para obtener y evaluar la evidencia disponible perteneciente a un tema específico [14]. RS es el proceso de 
resumir información existente sobre un fenómeno de forma minuciosa y empírica. Al final, la RS obtiene como resultado una conclusión general sobre los estudios individuales del fenómeno en cuestión.

\section{B. El proceso de la $R S$.}

En este apartado se describe de manera general en 3 pasos, el proceso de la revisión sistemática para el área de Ingeniería de Software, propuesto por Biolchini et al. [15].

1) Desarrollo del protocolo: fase rigurosa e iterativa. Cubre el plan general para la revisión sistemática de la literatura.

a) Formulación de la pregunta: sección donde se identifican las preguntas de investigación importantes que deben ser contestadas durante la RS. En esta sección los objetivos de la investigación deben estar claramente definidos. Se compone de los siguientes apartados: enfoque de la pregunta, amplitud y calidad de la pregunta.

b) Identificación y selección de fuentes: sección cuyo objetivo es identificar y seleccionar las fuentes donde se llevará a cabo la búsqueda de los estudios primarios. Se compone de los siguientes apartados: definición de los criterios de la selección de fuentes, idioma de los estudios, identificación de fuentes, selección de fuentes después de la evaluación, verificación de las fuentes.

c) Selección de estudios: sección que tiene por objetivo identificar los estudios primarios y secundarios. Los estudios se seleccionan después de la aplicación de los criterios de inclusión y exclusión.

2) Extracción de información: fase en la que se ejecuta la búsqueda de estudios en las fuentes definidas, los estudios encontrados se evalúan con los criterios definidos.

a) Definición de los criterios de inclusión de información (ICinf) y exclusión de información (ECinf), que tiene por objetivo definir los criterios con que será evaluada la información.

b) Plantillas para la extracción de información: sección que tiene por objetivo registrar los estudios primarios derivados del proceso de selección.

c) Ejecución de la extracción: sección en la que se realiza la evaluación de los estudios mediante los criterios definidos

3) Resumen de resultados: fase en la que se muestran los resultados obtenidos de la aplicación de la fase de desarrollo del protocolo de RS y la fase de extracción de información.

a) Tendencia de los estudios: sección que muestra cómo han evolucionado los estudios primarios a lo largo del tiempo.

b) Clasificación: sección que muestra los resultados encontrados en los estudios primarios al aplicar los criterios de inclusión y exclusión de la información.

\section{DESARROLLO DEL PROTOCOLO}

En esta sección se describen los pasos realizados para el desarrollo del protocolo para revisiones sistemáticas. En el apartado A, se establece el enfoque, amplitud y calidad de la pregunta. En el apartado B, se definen los criterios de la selección de fuentes, se define el idioma de los estudios y se identifican las fuentes. En el apartado C, se definen los criterios de inclusión y exclusión de estudios y se realiza la selección.

\section{A. Formulación de la pregunta:}

1) Enfoque de la pregunta: esta RS se lleva a cabo para identificar iniciativas o informes de experiencias en modelos, metodologías, estándares y procesos de soporte del software del área de calidad en las MiPyMEs, así como para dar a conocer las tendencias, países, y sectores con mayor número de publicaciones en este ámbito.

2) Amplitud y calidad de la Pregunta: esta sección ayuda a definir la sintaxis de la pregunta de investigación, el contexto en el cual se aplica la revisión y la pregunta de investigación a ser contestada, así como sus especificaciones semánticas (o rango de la pregunta).

Problema: un marco de trabajo para la mejora de procesos software es un mecanismo eficaz para la mejora de la calidad del software [16], debido a que ayuda a elaborar software de forma sistemática y cuantificable [10]. Los procesos de soporte son procesos definidos dentro del grupo de procesos del ciclo de vida del software. Tales procesos de soporte desempeñan un rol integral en asistir a los procesos de implementación del software [17]. En la actualidad no existen estudios recientes que muestren las tendencias de las iniciativas del tópico seleccionado, por lo que creemos que es de interés dar a conocer qué países y en qué sectores se llevan a cabo los estudios, así como los modelos, metodologías, estándares, y procesos de soporte del software del área de calidad utilizados en las MiPyMEs. A continuación se describe la pregunta de la RS, la intervención, el efecto, los resultados de las mediciones, la población y la aplicación.

3) Pregunta: ¿Qué modelos, métodos, estándares y procesos de soporte del software del área de calidad se abordan en las adaptaciones SPI para las MiPyMEs desarrolladoras de software?.

4) Intervención: marcos de trabajo para la mejora de procesos en MiPyMEs desarrolladoras de software.

5) Efecto: iniciativas que tratan modelos, métodos, estándares y procesos de soporte del software del área de calidad para MiPyMEs desarrolladoras de software.

6) Resultados de las mediciones: número de iniciativas identificadas.

7) Población: publicaciones relacionadas con modelos, métodos, estándares, y procesos de soporte del software del área de calidad en el contexto de las MiPyMEs.

8) Aplicación: organizaciones que emplean algún marco de trabajo para la mejora de procesos en MiPyMEs desarrolladoras de software.

\section{B. Identificación y selección de fuentes:}

El objetivo de este apartado es identificar y seleccionar las fuentes donde se ejecutará la búsqueda de los estudios primarios [14].

1) Definición de los criterios de la selección de fuentes:

- Utilizar mecanismos de búsqueda con palabras clave y sitios sugeridos por expertos.

- Publicaciones recomendadas por expertos.

- Publicaciones disponibles en sitios web.

2) Idioma de los estudios:

- Inglés.

3) Identificación de fuentes: 
Las fuentes incluyen las bases de datos digitales: ACM, CiteeSeerX, IEEE Xplore, Sciencie Direct, Wiley y SEI.

Cadenas de búsqueda: palabras reservadas extraídas del conjunto de palabras definidas en la pregunta. Combinando estas palabras clave con los operadores lógicos "AND" y "OR", se obtuvo la cadena de búsqueda (que se muestra en la Tabla 2). Esta cadena ha sido adaptada a cada buscador de las fuentes de búsqueda.

\section{TABLA 2.CADENA DE BÚSQUEDA.}

\section{Palabras clave con operadores lógicos}

("software process improvement" or SPI) AND (small or "medium" or "small and medium" or "SME's") AND (settings or organizations or companies or company or "sized software company" or enterprises or "software enterprises")

\section{4) Selección de fuentes después de la evaluación de criterios:}

Se verificó, si las fuentes se ajustan a los criterios previamente definidos, y se estableció la lista de fuentes, mostrada en la Tabla 3.

TABLA 3. FUENTES EMPLEADAS.

\begin{tabular}{|c|c|}
\hline Número & Fuentes \\
\hline 1 & ACM \\
\hline 2 & CiteeSeerX \\
\hline 3 & IEEE Xplore \\
\hline 4 & Science Direct \\
\hline 5 & Wiley \\
\hline 6 & SEI \\
\hline
\end{tabular}

\section{5) Verificación de las fuentes:}

Tres investigadores del Grupo de Investigación en Ingeniería de Software y Software Educativo, de la Facultad de Matemáticas en la Universidad Autónoma de Yucatán evaluaron el listado de fuentes, de acuerdo con los criterios de selección de fuentes, obtenida en la sección previa, donde de forma consensuada aprobaron el listado.

\section{Selección de estudios:}

Este apartado describe el proceso y criterios de la selección y evaluación de los estudios.

\section{1) Definición de estudios:}

En la definición de criterios de inclusión (CI) de estudios y de exclusión (CE) de estudios [14], se identificaron 4 y 2, respectivamente, los cuales se muestran en la Tabla 4.

TABLA 4. DEFINICIÓN DE CRITERIOS DE INCLUSIÓN Y EXCLUSIÓN DE ESTUDIOS.

\begin{tabular}{|c|l|}
\hline Criterio & \multicolumn{1}{|c|}{ Descripción } \\
\hline CI1 & $\begin{array}{l}\text { Incluye publicaciones cuyos títulos están relacionados } \\
\text { con la mejora de proceso software en las MiPyMEs. }\end{array}$ \\
\hline CI2 & $\begin{array}{l}\text { Incluye publicaciones que contengan palabras reservadas } \\
\text { que coincidan con las definidas en la cadena de } \\
\text { búsqueda. }\end{array}$ \\
\hline CI3 & $\begin{array}{l}\text { Incluye publicaciones cuyo resumen esté relacionado con } \\
\text { el tópico seleccionado. }\end{array}$ \\
\hline CI4 & $\begin{array}{l}\text { Incluye publicaciones que han sido leídas parcial o } \\
\text { totalmente. }\end{array}$ \\
\hline CE1 & $\begin{array}{l}\text { Excluye publicaciones que no coinciden con los criterios } \\
\text { de inclusión previos. }\end{array}$ \\
\hline CE2 & Excluye tolas las publicaciones duplicadas. \\
\hline
\end{tabular}

Definición de tipos de estudios: se analizaron los estudios relacionados con la mejora de procesos software en las MiPyMEs.

Con respecto al procedimiento para seleccionar los estudios, se tomaron como criterios: el título de la publicación, el resumen de cada uno y en su mayoría se requirió revisar el contenido completo.

\section{2) Ejecución de la selección:}

Selección inicial de estudios: se realizó la ejecución de las búsquedas, adaptando las cadenas a los motores de cada buscador. La Tabla 5 muestra los resultados obtenidos.

Evaluación de la calidad de los estudios: para determinar la calidad de los estudios, se aplicaron los criterios de inclusión y de exclusión, donde se seleccionaron 50 estudios.

TABLA 5. NÚMERO DE ESTUDIOS Y RESULTADOS DE LAS BÚSQUEDAS.

\begin{tabular}{|l|c|c|c|}
\hline \multicolumn{1}{|c|}{ Fuente } & $\begin{array}{c}\text { Fecha } \\
\text { Búsqueda }\end{array}$ & Resultados & $\begin{array}{c}\text { Estudios } \\
\text { Primarios }\end{array}$ \\
\hline ACM & $24 / 06 / 2013$ & 79 & 6 \\
\hline CiteeSeerX & $25 / 06 / 2013$ & 89 & 10 \\
\hline IEEE Xplore & $24 / 06 / 2013$ & 47 & 13 \\
\hline Science Direct & $15 / 01 / 2014$ & 191 & 14 \\
\hline Wiley & $26 / 06 / 2013$ & 426 & 5 \\
\hline SEI Total & $26 / 06 / 2013$ & 82 & 2 \\
\hline \multicolumn{2}{r|}{} & $\mathbf{9 0 7}$ & $\mathbf{5 0}$ \\
\hline
\end{tabular}

\section{EXTRACCIÓN DE INFORMACIÓN}

En esta sección se describen los pasos realizados para la extracción de información, en los estudios primarios encontrados. Primero se definieron los criterios de inclusión de información (Clinf) y exclusión de información (CEinf). En segundo lugar se elaboraron plantillas para el registro de los datos extraídos, por último, se ejecutó la extracción de la información aplicando los CIinf, y los CEinf.

\section{A. Definición de los criterios de inclusión de información (CIinf) y exclusión de información (CEinf).}

Respecto a este punto, se identificaron cuatro criterios de inclusión de información (Clinf) y uno de exclusión (CEinf). La Tabla 6 muestra una descripción de estos criterios.

TABLA 6. DEFINICIÓN DE LOS CRITERIOS DE INCLUSIÓN Y EXCLUSIÓN DE INFORMACIÓN.

\begin{tabular}{|c|l|}
\hline Criterio & \multicolumn{1}{c|}{ Descripción } \\
\hline CI1inf & $\begin{array}{l}\text { Recoger información sobre las tendencias en investigación e } \\
\text { implementación respecto a la mejora de procesos software } \\
\text { en las MiPyMEs. }\end{array}$ \\
\hline CI2inf & $\begin{array}{l}\text { Recoger información sobre países y sectores que investigan } \\
\text { o implementan alguna mejora de los procesos software en } \\
\text { las MiPyMEs. }\end{array}$ \\
\hline CI3inf & $\begin{array}{l}\text { Identificar qué modelos, metodologías y estándares abordan } \\
\text { los estudios de mejora del proceso de soporte en las } \\
\text { MiPyMEs. }\end{array}$ \\
\hline CI4inf & $\begin{array}{l}\text { Identificar qué proceso de soporte del área de la calidad del } \\
\text { ciclo de vida del software tratan los estudios. }\end{array}$ \\
\hline CE1inf & $\begin{array}{l}\text { Excluir la información que no esté relacionada con los } \\
\text { criterios de inclusión definidos anteriormente. }\end{array}$ \\
\hline
\end{tabular}

\section{B. Plantillas para la Extracción de Información}

Para el registro de los datos de identificación del estudio primario, se definieron plantillas que contienen los siguientes campos: identificador (id), año, y título del estudio. Para el registro de los datos a extraer mediante los criterios CIinf y los CEinf, se definieron plantillas con los siguientes campos: resumen, país, institución u organización, metodología, modelo o estándar y aspecto de mejora. Los datos registrados en estas plantillas se usaron para el apartado de resumen de resultados.

\section{Ejecución de la Extracción}

Con una evaluación imparcial de la información de los estudios primarios, los registros obtenidos se colocaron en las plantillas para la extracción de la información. La evaluación se realizó mediante observaciones de las ideas principales, 
utilizando los criterios de inclusión y exclusión de la información (véase Tabla 6) y se organizó de acuerdo a los criterios de inclusión.

\section{RESUMEN DE RESULTADOS}

En esta sección se presentan los resultados obtenidos de la aplicación del protocolo de la RS.

\section{A. Tendencia de los estudios}

En el análisis de tendencia de los estudios, se consideró el año del estudio. Como se muestra en la Figura 1, los estudios tienen una tendencia incremental. Los años donde se tuvieron más estudios sobre el tópico seleccionado fueron 2010 y 2013. Estos resultados sugieren interés del tópico en la comunidad, en aportar al cuerpo de conocimiento de Ingeniería de Software, aspectos relacionados a la mejora de procesos para las MiPyME's. Estos resultados corroboran los resultados publicados en [10].

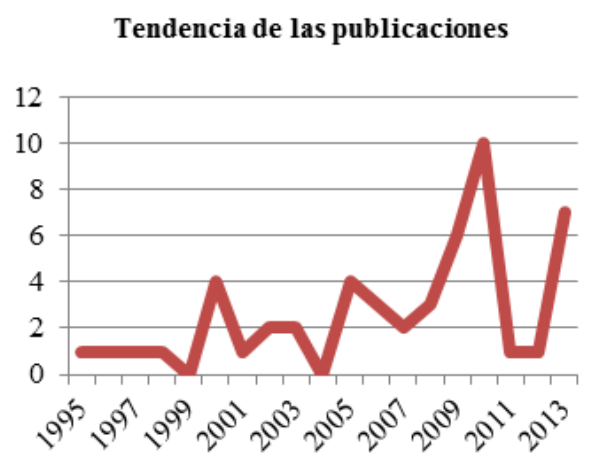

FIGURA 1.PUBLICACIONES DEL TÓPICO POR AÑO.

\section{B. Clasificación}

Los estudios cubren:

1) Países que han publicado sobre el tópico seleccionado:

En el análisis de los países que publican, se identificó el país del autor principal del estudio. Como se muestra en la Figura 2, el $46 \%$ corresponde a 6 países, que son: España con el 10\%, Estados Unidos, Brasil e Irlanda con el $8 \%$, respectivamente, Bélgica y Colombia con el $6 \%$, respectivamente. El 54\% restante corresponde a otros países: Alemania, Arabia, Austria, Australia, Canadá, Chile, China, Corea del Sur, Dinamarca, Finlandia, Francia, India, Italia, Japón, México, Noruega, Pakistán, Reino Unido y Suiza. Estos resultados sugieren que el tópico seleccionado es de interés a nivel mundial, principalmente en países desarrollados y emergentes. Éste interés muy probablemente se deba a la dependencia que hay entre las economías mundiales y las MiPyMEs de este sector (representadas por el 99\% [2]), así como a la existencia de programas de apoyo gubernamentales tales como SPIRE (Software Process Improvement in Regions of Europe) [18], "mps br" (melhoria do proceso de software brasileiro) [19], SIMEP-SW (Sistema Integral para la Mejora de los Procesos Software en Colombia) [20], COMPETISOFT (Mejora de Procesos para Fomentar la Competitividad de la Pequeña y Mediana Industria del Software de Iberoamérica) [21], MoProSoft (Modelo de Procesos para la Industria de Software en México) [22], entre otros.

\section{2) Resultados por tipos de sectores:}

En el análisis del tipo de sectores que publican, se consideró como sector educativo a las organizaciones que se dedican a la educación superior (universidades, academias, escuelas, institutos y facultades). Como centros de investigación, aquellas organizaciones que se dedican a la generación de conocimiento y su aplicación; y finalmente se consideró como sector privado a aquellas organizaciones que tienen como fin un beneficio económico y que no pertenecen al sector educativo, ni a un centro de investigación. En el caso de los centros de investigación, adscritos a una organización educativa, los estudios se catalogaron como producidos por el centro de investigación. Como se aprecia en la Figura 3, el sector que más publica es el sector educativo con un $84 \%$, seguido del centro de investigación con $10 \%$ y finalmente el sector privado con 6\%. Estos resultados sugieren que las aportaciones se mantienen en el ámbito conceptual y que es escasa la vinculación con el sector privado, lo que se corrobora de acuerdo con los resultados publicados en [23].

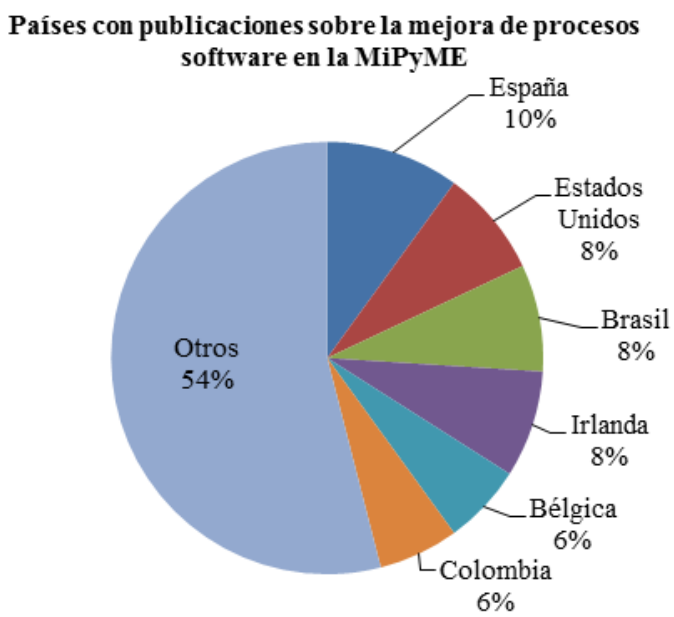

FiguRA 2. PAÍSES CON PUBLICACIONES SOBRE EL TÓPICO SELECCIONADO.
Sectores que publican sobre la mejora de procesos software en la MiPyME

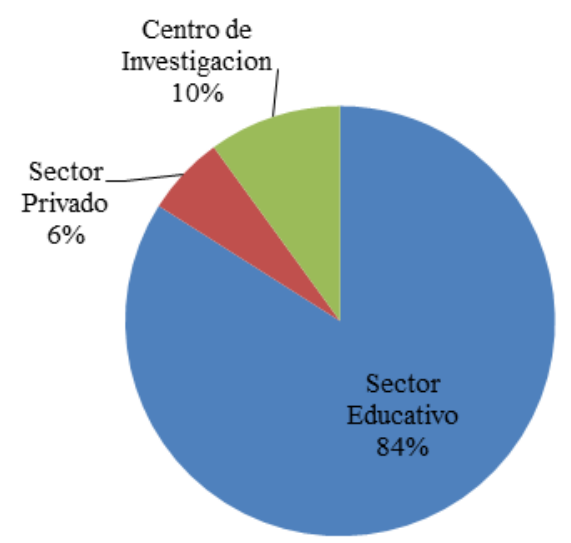

FIGURA 3. SECTORES QUE PUBLICAN EN EL TÓPICO SELECCIONADO.

\section{3) Modelos de proceso usados:}

En el análisis de los modelos usados, se tomaron en cuenta aquellos estudios que se basaron, cuando menos, en algún modelo (CMMi [7], CMM [5], SPICE [24], Otros [25] - [33]). En los casos donde los estudios se apoyaron en más de un modelo, éstos últimos se separaron y se clasificaron en la categoría correspondiente. Como se observa en la Figura 4, el modelo más usado es CMMi [7] con un 40\%, seguido por SPICE [24] con $21 \%$ y por CMM [5] con un 17\%. Así mismo se observa que existen otros modelos que en su conjunto suman el 22\%: BOOTSTRAP [25], IDEAL [26], PDCA [27], MESOPYME [28], MDE [29], TQM [30], KMDL-Procedural Model [31], Micro-Evaluation Model [32], PRIMER [33]. Estos resultados muestran que los modelos de calidad tratados en los 
estudios revisados, son adaptaciones que se basan principalmente en los modelos para las grandes empresas. Aun cuando existen otros modelos de calidad para la MiPyME. Esto sugiere cierto grado de inmadurez en el conocimiento, la aceptación y la experimentación de dichos modelos en las MiPyMEs.

\section{Modelos de proceso usados}

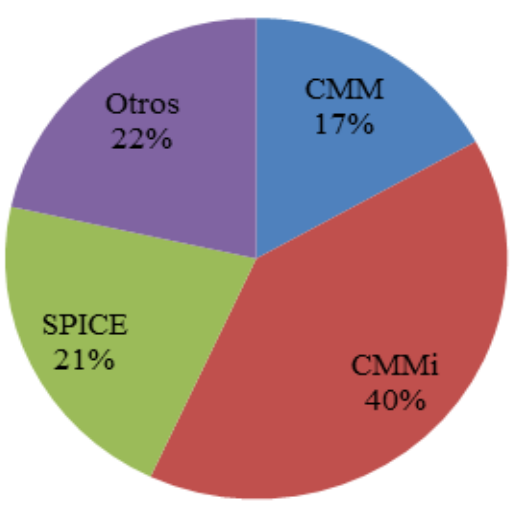

FIGURA 4. MODELOS DE PROCESO USADOS.

\section{4) Metodologías consideradas.}

En el análisis de las metodologías utilizadas, se consideraron los estudios que trataron, al menos, alguna metodología (XP [34], RAPID [35], Otras [36]-[40]). Si alguna publicación consideró más de una, éstas se separaron y se clasificaron en la categoría correspondiente. Como se muestra en la Figura 5, el 54\% de las metodologías se concentran en Xtreme Programing (XP) [34] y RAPID [35] con 36\% y 18\%, respectivamente. El 46\% restante concentra a las otras métodologías: PIPSI [36], QFD [37], METvalCOM-PETISOFT [38], SCRUM [39], DSDM [40]. Estos resultados sugieren que XP [34] es una las metodologías más usadas, lo que se corrobora de acuerdo con los resultados publicados en [11]. Por otra parte, se observa que RAPID [35] está ganando interés como lo muestran publicaciones previas [10].

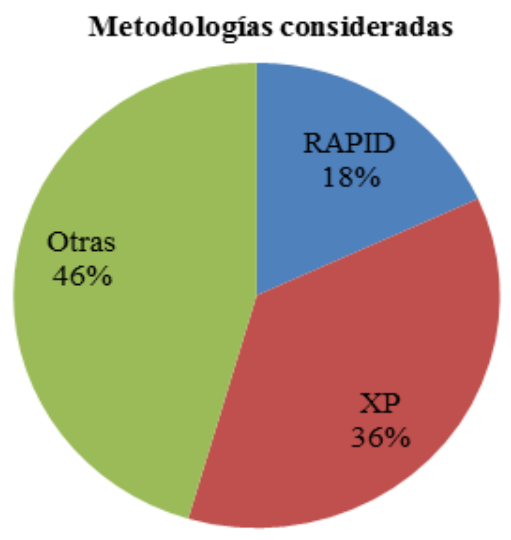

Figura 5. METODOLOGÍAS CONSIDERADAS.

\section{5) Estándares utlizados:}

En el análisis de los estándares empleados, se consideraron los estudios que trataron, al menos, algún estándar. Éstos se clasificaron en su categoría correspondiente (ISO/IEC 15504 [6], ISO 9000 [41], ISO9001 [42], ISO/IEC 12207[17], ISO/IEC 17021 [43]). Como se muestra en la Figura 6, el estándar de mayor uso es ISO/IEC 15504 [6] con un 52\%, seguido por ISO 9000 con un $20 \%$, por ISO 9001 [42] con un $16 \%$, por ISO/IEC 12207 [17] con un $8 \%$, y finalmente por
ISO/IEC 17021 [43] con un 4\%. Estos resultados sugieren que la comunidad en Ingeniería de Software está apostando a estándares ISO, ocasionando que sean conocidos entre la comunidad y no tan complicados en su implementación, además de ofrecer la posibilidad de obtener una certificación internacional [44][47].

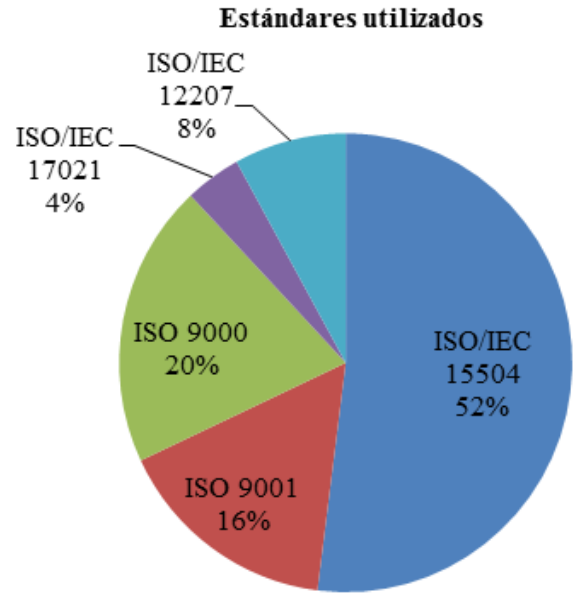

FIGURA 6. ESTÁNDARES UTILIZADOS.

\section{6) Procesos de soporte del software del área de calidad:}

En el análisis de los procesos de soporte del área de calidad del ciclo de vida, se seleccionaron los estudios que aportaron alguna mejora, al menos, en uno de los procesos de soporte mencionados. En los casos donde las publicaciones aportaron alguna mejora en más de un proceso de soporte del software, éstos últimos se separaron y se clasificaron en las categorías correspondientes (aseguramiento de la calidad, verificación, validación y auditoria). Como se observa en la Figura 7, los procesos de soporte del área de calidad del software que reciben contribuciones de los estudios seleccionados son: aseguramiento de la calidad con un $46 \%$, verificación con un $41 \%$, validación con $11 \%$, y auditoria con $2 \%$. Estos resultados sugieren que la comunidad de Ingeniería de Software está priorizando la formalización y la verificación de los procesos que ayuden a asegurar la calidad del software, así como la formalización de las validaciones de los productos software [10]. Algunas razones que quizá está potenciando la investígación en dichos procesos son las especificaciones incompletas, cambios continuos en requerimientos [48], falta de formalidad en procesos y metodologías [49], así como la carencia de control en los procesos $[50,51]$, problemas comunes en la MiPyMEs desarrolladoras de software.

\section{Procesos de soporte del software delárea de calidad}

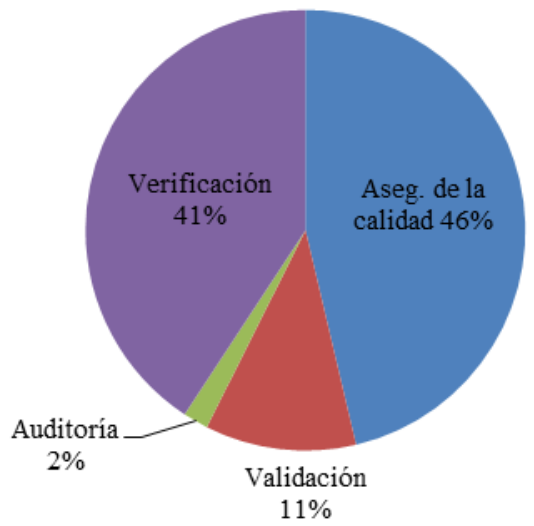

Figura 7. PRocesos DE SOPORTE DEL SOFTWARE DEL ÁREA DE CALIDAD. 


\section{CONCLUSIONES}

Las revisiones sistemáticas permiten sintetizar la información existente sobre un fenómeno de forma minuciosa y empírica. Al final, la revisión sistemática obtiene como resultado una conclusión general sobre los estudios individuales del fenómeno en cuestión.

En este trabajo se han presentado los resultados de una revisión sistemática de la literatura sobre las contribuciones a la mejora de procesos software del área de calidad, en el contexto de las MiPyME's desarrolladoras de software, en el periodo comprendido de 1995 a diciembre de 2013.

El interés de la comunidad de Ingeniería de Software, en relación al tópico seleccionado, muestra una tendencia incremental que sugiere continuará. Se encontró que a nivel mundial, principalmente en los países desarrollados y emergentes, la mayoría de estudios se han realizado en el sector educativo y en los centros de investigación, quedando relegado el sector privado. Estos resultados probablemente indiquen que la mayoría de las contribuciones del tema sean a nivel conceptual, quedando aún camino por recorrer referente a las aportaciones del sector privado.

En cuanto a modelos y metodologías desarrolladas para la MiPyME, los modelos preferidos para su adaptación son: CMM [5], CMMi [7], y SPICE [24]. Por otra parte, las metodologías más usadas son: Extreme Programming [34] y RAPID [35]. Respecto a los estándares preferidos para su adaptación a la MiPyME, se encuentran: ISO/IEC 15504 [6], ISO 9000 [41] e ISO 9001 [42].

También se encontró que los esfuerzos están centrados en la formalización y la verificación de los procesos, así como la formalización de las validaciones de los productos software, que ayudan en el aseguramiento de la calidad del software.

Se espera que estos resultados contribuyan al cuerpo de conocimientos del tema en cuestión y motive mayor investigación.

\section{REFERENCIAS}

[1] J. Banegas, "Informe AETIC 2007," Instituto Español de Comercio Exterior, Madrid, España.2008.

[2] M. E. Fayad, M. Laitinen, and R. P. Ward, "Software Engineering in the Small," Communications of the ACM, pp. 123-132, 2000.

[3] D. Jeans-Marcc, Z. Mohammad, and A. Alain, "Very Small Enterprises (VSE) Quality Process Assesment," 2007.

[4] S. F. Ochoa, A. Quispe, A. Vergara, and J. A. Pino, "Improving Requirements Engineering Processes in Very Small Software Enterprises Through the Use of a Collaborative Application," in 14th International Conference on Computer Supported Cooperative Work in Design, 2010.

[5] Paulk. Mark, Curtis. William, Chrissis. Mary Beth, and W. Charles, "Capability Maturity Model for Software," Software Engineering Institute, Carnegie Mellon University, Pittsburgh, Pennsylvania, 1993.

[6] I. S. Organization, "ISO/IEC 15504-2:2003, Information technology — Process assessment — Part 2: Performing an assessment," vol. ISO/IEC 15504-2:2003, ed: International Standar Organization, 2004.

[7] C. P. Team, "CMMI for Development, Version 1.3 CMU/SEI2010-TR-033," Carnegie Mellon University - SEI, Pittsburgh, PA2010.

[8] T. Grechenig and W. Zuser, "Creating organic software maturity attitudes (COSMA) selected principles and activities for software maturity in small and medium software enterprises," in Fourth International Conference on Quality Software, 2004, pp. 134-143.
[9] I. Richardson, "Software process matrix: a small company SPI model. Software Process: Improvement and Practice," vol. 6, pp. 157-165, 2001.

[10] Francisco J. Pino, Félix García, and M. Piattini. (2006) Revisión sistemática de mejora de procesos software en micro, pequeñas y medianas empresas. Revista Española de Innovación, Calidad e Ingeniería del Software. 6-23.

[11] T. Dybå and T. Dingsøyr, "Empirical studies of agile software development: A systematic review," Information and Software Technology, vol. 50, pp. 833-859, 8// 2008.

[12] Christiane Gresse von Wangenheim, Jean Carlo Rossa Hauck, Clenio F. Salviano, and A. v. Wangenheim, "Systematic Literature Review of Software Process Capability/Maturity Models," in International Conference on Software Process. I, Pisa, Italy, 2010.

[13] D. Brenda, M. Mirna, and M. Jezreel, "Actual state of implementing software process improvements in software organizations," presented at the Iberian Conference on Information Systems and Technologies (CISTI), 20132013.

[14] Preliminary Guidelines for Emprical Research in Software Enginerring, N. R. C. o. Canada, 2001.

[15] J. Biolchine, P. Gomes, A. Cruz, and G. Orta, "Systematic review in software engineering," Rio de Janeiro RT-ES679/05, 2005.

[16] M. A. C. Contreras, "Desarrollo e implementación de un framework par la formación de carteras de proyectos de I\&D en organizaciones públicas," Maestria, Universidad Autónoma de Nuevo León, 2007.

[17] ISO/IEC/IEEE, "IEEE STD 12207-2008 Standard for Systems and Software Engineering - Software Life Cycle Processes," ed, 2008.

[18] S. P. T. C. f. S. Engineering, "Software Process Improvement in Regions of Europe (SPIRE)," 1993.

[19] K. Weber, E. Araújo, A. Rocha, Machado, D. Scalet, and C. Salviano. (2004) Brazilian Software Process Reference Model and Assessment Method. Computer and Infomation Sciences. 402-411.

[20] J. A. Hurtado and C. Bastarrica, "PROYECTO SIMEP-SW," Universidad del Cauca2005.

[21] E. d. P. COMPETISOFT, "COMPETISOFT - Mejora de Procesos para Fomentar la Competitividad de la Pequeña y Mediana Industria del Software de Iberoamérica. Versión 0.2," 2006.

[22] H. Oktaba, C. A. Esquivel, A. S. Ramos, A. M. Martínez, G. Q. Osorio, M. R. López, et al., "Modelo de Procesos para la Industria de Software MoProSoft," ed: Versión, 2003.

[23] J. M. Vega Jurado, I. Fernández de Lucio, and R. Huanca López, "La Relación Universidad-Empresa en América Latina: ¿Apropiación Incorrecta de Modelos Foráneos?," 2007, vol. 2, 2007.

[24] I. O. f. Standardization, "ISO/IEC TR 15504:1998(E)," in Information Technology -Software process assessment, ed. Geneva: ISO/IEC, 1998.

[25] V. Haase, R. Messnarz, G. Koch, H. J. Kugler, and P. Decrinis, "Bootstrap: fine-tuning process assessment," Software, IEEE, vol. 11, pp. 25-35, 1994.

[26] M. Robert, IDEAL: A User's Guide for Software Process Improvement. Pittsburgh, Pennsylvania: Software Engineering Institute, 1996.

[27] M. Imai, Kaizen: The Key to Japan's Competitive Success: McGraw-Hill Higher Education, 1988.

[28] J. Calvo-Manzano Villalón, G. Cuevas Agustín, T. San Feliu Gilabert, A. De Amescua Seco, L. García Sánchez, and M. Pérez Cota, "Experiences in the Application of Software Process Improvement in SMES," Software Quality Journal, vol. 10, pp. 261-273, 2002/11/01 2002. 
[29] C. S. Douglas. (2006) Guest Editor's Introduction: ModelDriven Engineering. 25-31. Available: http://doi.ieeecomputersociety.org/10.1109/MC.2006.58

[30] W. E. Deming, Out of the Crisis. Cambridge, Massachusetts: Massachusetts Institute of Technology, 1986.

[31] N. Gronau, C. Müller, and R. Korf, "KMDL - Capturing, Analysing and Improving Knowledge-Intensive Business Processes," Journal of Universal Computer Science, vol. 11, pp. 452--472, 2005.

[32] N. Habra, S. Alexandre, J.-M. Desharnais, C. Y. Laporte, and A. Renault, "Initiating software process improvement in very small enterprises: Experience with a light assessment tool," Information and Software Technology, vol. 50, pp. 763-771, 6// 2008.

[33] J. Karjalainen, M. Mäkäräinen, S. Komi-Sirviö, and V. Seppänen, "Practical Process Improvement for Embedded RealTime Software," Quality Engineering, vol. 8, pp. 565-573, 1996/06/01 1996.

[34] K. Beck, Extreme programming explained: Embrace change. Boston: addition-Wesley, 2000.

[35] T. Rout, A. Tuffley, B. Cahill, and B. Hodgen, "The rapid assessment of software process capability," presented at the First International Conference on Software Process Improvement and Capability Determination, Limerick, 2000.

[36] Rory O'Connor, Howard Duncan, Gerry Coleman, Maurizio Morisio, Cliona McGowan, Christophe Mercier, et al., "Improving Professional Software Skills in Industry - A Training Experiment," Dublin City University, Dublin2001.

[37] Y. Akao, Development History of Quality Function Deployment. Minato, Tokyo Asian Productivity Organization, 1994.

[38] F. J. Pino, C. Pardo, F. García, and M. Piattini, "Assessment methodology for software process improvement in small organizations," Information and Software Technology, vol. 52, pp. 1044-1061, 10// 2010.

[39] K. Schwaber, "The scrum development process," presented at the Proceedings of the Tenth Annual Conference on ObjectOriented Programming Systems, Languages, and Applications, Austin, Texas, USA, 1995.

[40] B. J. J. Voigt, "Dynamic System Development Method," Department of information technology university of Zurich, Zurich, 2004.

[41] I. O. f. Standardization, "ISO 9000 Quality management and quality assurance standards," in Guidelines for selection and use ed. Geneva: ISO, 1994.

[42] I. O. f. Standardization, "ISO 9001 Quality systems. ," in Model for quality assurance in design, development, production, installation and servicing, ed. Geneva: ISO, 1994.

[43] I. O. f. Standardization, "ISO/IEC 17021:2011. Conformity assessment," in Requirements for bodies providing audit and certification of management systems, ed: ISO, 2011.

[44] A. Cater-Steel, M. Toleman, and T. Rout, "Process improvement for small firms: An evaluation of the RAPID assessment-based method," Information and Software Technology, vol. 48, pp. 323-334, 2006.

[45] A. Mas, B. Fluxà, and E. Amengual, "Lessons learned from an ISO/IEC 15504 SPI programme in a company," Journal of Software: Evolution and Process, vol. 24, pp. 493-500, 2012.

[46] J. Taramaa, M. Makarainen, and T. Ketola, "Improving Application Management Process through Qualitative Framework," in International Conference on Software Maintenance, 1995, pp. 327-336.

[47] Gonzalo Valdés, Marcello Visconti, and H. Astudillo, "The Tutelkan Reference Process: A Reusable Process Model for Enabling SPI in Small Settings," presented at the EuroSPI, 2011.
[48] Quispe, M. Marques, L. Silvestre, S. Ochoa, and R. Robbes, "Requirements Engineering Practices in Very Small Software Enterprises: A Diagnostic Study," in XXIX International Conference of the Chilean Computer Science Society, 2010.

[49] S. Alexandre, A. Renault, and N. Habra, "OWPL: A Gradual Approach for Software Process Improvement In SMEs," in 32nd EUROMICRO Conference on Software Engineering and Advanced Applications, 2006.

[50] C. Hofer, "Software Development in Austria: Results of an Empirical Study among Small and Very Small Enterprises," presented at the 28 th Euromicro Conference 2002.

[51] F. J. Pino, O. Pedreira, F. Garcia, M. Rodriguez, and M. Piattini, "Using Scrum to the Guide the Execution of Software Process Improvement in Small Organization," The Journal of System and Software, 2010.

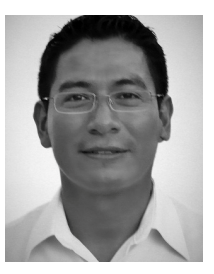

Gerzon E. Gómez es Ingeniero en Sistemas Computacionales por la Universidad Autónoma de Tamaulipas en el año 1999. Doctor candidato en Informática por la Universidad Politécnica de Madrid (UPM). Profesor en el área de Ingeniería de Software en la Facultad de Matemáticas de la Universidad Autónoma de Yucatán (UADY). Su área de investigación se centra en la Ingeniería de Software. Es colaborador en el grupo de investigación SEMEPRO (Seguridad y Mejora de Procesos) y en el proyecto "Cátedra de Mejora de Procesos Software en el Espacio Iberoamericano" (MPSEI) de la UPM.

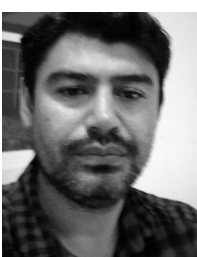

Antonio A. Aguileta es Licenciado en Ciencias de la Computación por la Universidad Autónoma de Yucatán (UADY), Maestro en Ciencias Computacionales por el Instituto Tecnológico y de Estudios Superiores de Monterrey (ITESM), campus Monterrey. Actualmente es Profesor Asociado en la Facultad de Matemáticas de la UADY. La línea de investigación de su interés es en torno a la calidad en la Ingeniería de Software.

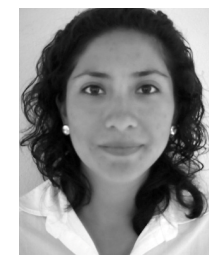

Grisel B. Ancona es estudiante del octavo semestre de la carrera de Ingeniería de Software en la Facultad de Matemáticas de la Universidad Autónoma de Yucatán. El trabajo aquí reportado corresponde con una parte de su tesis de pregrado titulada: Avances de marcos de trabajo de desarrollo y mejora de procesos software. Áreas de interés: mejora de procesos software, calidad del software, administración de proyectos software.

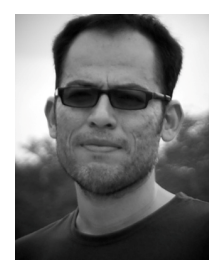

Omar S. Gómez es Ingeniero en Computación por la Universidad de Guadalajara (México, 2001), Maestro en Ingeniería de Software por el Centro de Investigación en Matemáticas (México, 2005), Doctor en Software y Sistemas por la Universidad Politécnica de Madrid (España, 2012). Actualmente es colaborador del Grupo de Investigación en Ingeniería de Software de la Facultad de Matemáticas en la Universidad Autónoma de Yucatán. Su área de investigación se centra en Ingeniería de Software Empírica. 